\title{
ARTICLE
}

Stem cell transplantation

\section{What is the best first-line treatment for POEMS syndrome: autologous transplantation, melphalan and dexamethasone, or lenalidomide and dexamethasone?}

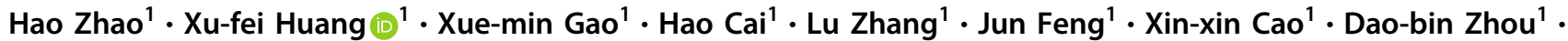 \\ Jian Li (iD ${ }^{1}$
}

Received: 18 October 2018 / Revised: 23 December 2018 / Accepted: 7 January 2019 / Published online: 30 January 2019

(c) The Author(s) 2019. This article is published with open access

\begin{abstract}
POEMS syndrome is a rare plasma cell dyscrasia. This study compared the responses to and survival of 347 POEMS syndrome patients given three first-line treatment regimens: autologous stem cell transplantation (ASCT, $N=165$ ) and melphalan + dexamethasone (MDex, $N=79$ ), or lenalidomide + dexamethasone (LDex, $N=103$ ). After a median 45month follow-up, overall hematologic complete remission $\left(\mathrm{CR}_{H}\right)$ was $46.4 \%$, vascular endothelial growth factor complete remission $\left(\mathrm{CR}_{V}\right)$ was $55.1 \%$, and neurological remission $\left(\mathrm{R}_{N}\right)$ was $93.8 \% . \mathrm{CR}_{H}$ was better with ASCT $(49.7 \%)$ than with $\operatorname{MDex}(37.7 \%, p=0.001) . \mathrm{CR}_{V}$ was better with ASCT $(66.2 \%)$ than with $\operatorname{MDex}(38.5 \%, p=0.001)$ or LDex $(47.7 \%, p=$ 0.008). Differences in $\mathrm{R}_{N}$ achieved by three regimens $(91.5 \%$ vs. $100 \%$ vs. $93.8 \%, p=0.234)$ were not significant. Overall 3-year progression-free survival (PFS) was $80.5 \%$ and overall 3-year overall survival (OS) was 90.8\%. PFS was $87.6 \%$ with ASCT and $64.9 \%$ with LDex $(p=0.003)$. OS in the three regimens did not differ $(p=0.079)$. In medium-high risk patients, ASCT had better $\mathrm{CR}_{H}$ and $\mathrm{CR}_{V}$ than MDex, and better PFS than LDex. Therefore, although all three treatments had reasonable responses and survivals, patients with higher risk may benefit more from ASCT treatment.
\end{abstract}

\section{Introduction}

POEMS syndrome is a rare plasma cell dyscrasia characterized by polyneuropathy, organomegaly, endocrinopathy, monoclonal gammopathy, and skin changes. There is currently no standard treatment [1]. Current systematic therapies include high-dose chemotherapy with autologous stem cell transplantation (ASCT), alkylator-based therapy, and therapy with novel agents. High-dose chemotherapy with ASCT can achieve excellent clinical responses, with a significant improvement of neuropathy, complete hematologic response $\left(\mathrm{CR}_{H}\right)$ in $60 \%$ of POEMS syndrome patients, and complete

Supplementary information The online version of this article (https:// doi.org/10.1038/s41375-019-0391-2) contains supplementary material, which is available to authorized users.

$\triangle \mathrm{Jian} \mathrm{Li}$

lijian@pumch.cn

1 Department of Hematology, Peking Union Medical College Hospital, Chinese Academy of Medical Sciences and Peking Union Medical College, Beijing, China
VEGF response $\left(\mathrm{CR}_{V}\right)$ in 50\%. Five-year progression-free survival (PFS) of $75 \%$ and 5-year overall survival (OS) of $90 \%$ have been reported [2-4]. However, treatment-related complications are frequent, with peri-engraftment syndrome reported in 12-50\% of patients [5, 6]. Melphalan is an alkylation agent that is widely used for treatment of plasma disorders including myeloma and amyloidosis [7]. Melphalan plus dexamethasone (MDex) achieved 100\% neurological response $\left(\mathrm{R}_{N}\right), 38.7 \% \mathrm{CR}_{H}$, and $95.8 \% \mathrm{CR}_{V}$ in a prospective study of 31 POEMS syndrome patients, but long-term outcomes were not reported [8,9]. MDex is less toxic than ASCT, with no treatment-related mortality and a low incidence of severe adverse events. Lenalidomide is an immunomodulatory drug that is also cytotoxic to malignant plasma cells [10]. Lenalidomide plus dexamethasone (LDex) achieved an $\mathrm{R}_{N}$ of over $95 \%$, a $\mathrm{CR}_{H}$ of $46.3 \%$, a $\mathrm{CR}_{V}$ of $42.5 \%$, a 3-year PFS of $75 \%$, and an OS of $90 \%$ in a phase II single-arm prospective study including 41 patients [11]. All three treatments can achieve acceptable remission rates and survival, but it is unclear which is the best first-line treatment for POEMS syndrome. The efficacy of these treatments has not previously been compared in large patient cohorts, but the rarity of the disease makes it difficult to conduct randomized 
controlled trials. This study retrospectively evaluated the treatment efficacy and survival of the three treatment regimens in cohort of newly diagnosed POEMS syndrome patients treated at a single center.

\section{Patients and methods}

\section{Patients}

The medical records of POEMS syndrome patients admitted to our hospital between January 2000 and October 2017 and initially treated with ASCT, MDex, or LDex were evaluated. A total of 347 patients who satisfied the POEMS syndrome diagnosis criteria described by Dispenzieri et al. and had complete treatment and follow-up data were included [1]. All patients provided informed consent. The study was approved by the Institutional Review Board of Peking Union Medical College Hospital and conducted following the ethical guidelines of the Declaration of Helsinki.

\section{Risk stratification}

Patients were stratified to low, medium, or high risk groups using four baseline characteristics, age $>50$ years, presence of pulmonary hypertension, presence of pleural effusion, and an estimated glomerular filtration rate $<30 \mathrm{ml} / \mathrm{min} / 1.73$ $\mathrm{m}^{2}$. The first three characteristics had a value of 1 ; the last had a value of 2 . Patients with total scores 0,1 , and $2-5$ were assigned to low, medium, and high risk groups, respectively [12].

\section{Treatment regimens}

As no standard algorithm is available to guide the choice of treatment, the choice of regimen was mainly based on the clinician's judgment. Patients eligible for ASCT were $<65$ years of age, without serious systemic disease or organ dysfunction, severe pulmonary hypertension, severe capillary leak syndrome (hypotension and/or refractory ascites), and active infection, and with successful collection of adequate peripheral blood stem cells [13]. The protocol included mobilization with cyclophosphamide $3 \mathrm{~g} / \mathrm{m}^{2}$ and colony-stimulating factor and conditioning with high-dose melphalan $140-200 \mathrm{mg} / \mathrm{m}^{2}$. MDex therapy included melphalan $10 \mathrm{mg} / \mathrm{m}^{2}+$ dexamethasone $40 \mathrm{mg}$ on days $1-4$, one cycle every 28 days for a total of 9 cycles. LDex therapy included lenalidomide $10-25 \mathrm{mg}$ on days $1-21$, dexamethasone $40 \mathrm{mg}$ on days $1,8,15,22$, one cycle every 28 days for a total of 12 cycles. Prophylaxis of embolization with LDex therapy was by aspirin $100 \mathrm{mg}$ qd.

\section{Response criteria}

$\mathrm{CR}_{H}$ was assessed by immunofixation electrophoresis and free immunoglobulin light-chain negativity in both serum and urine. $\mathrm{CR}_{V}$ was defined as a decrease in VEGF to a normal concentration $(<600 \mathrm{pg} / \mathrm{ml})$. Achieving either $\mathrm{CR}_{H}$ or $\mathrm{CR}_{V}$ was defined as $\mathrm{CR}_{H} / \mathrm{CR}_{V}$. Neurological dysfunction was one of the key clinical manifestations and affects patients' life quality the most. Therefore, we use $\mathrm{R}_{N}$ to represent clinical remissions. $\mathrm{R}_{N}$ was defined as a decrease in the Overall Neuropathy Limitations Scale (ONLS) score of at least 1 point [8].

\section{Statistical analysis}

PFS was the time from treatment to recurrence, the deterioration of clinical symptoms, or death. OS was defined as the time from treatment to death. The date of last follow-up was August 31, 2018. Statistical analysis was performed with SPSS 22 (SPSS, Inc., Chicago, IL, USA). The chisquare test, or Fisher's exact test when appropriate, was used to determine the significance of differences in the values of categorical variables. Time to responses were calculated from the date of treatment initiation. Survival curves were plotted by the Kaplan-Meier method and differences were compared with the log-rank test. $p$-Values $<$ 0.05 were considered significant.

\section{Results}

\section{Patient characteristics}

The patient baseline characteristics are shown in Table 1. The median age at the time of diagnosis was 48 (range, 21-74) years; $65.7 \%$ were men. The median ONLS score was 4 of a possible $0-12$ points, and peripheral neuropathy was confirmed by electromyography in the 19 patients with ONLS scores of 0 . A total of $61.1 \%$ of the patients were positive for IgA type heavy chain monoclonal immunoglobulin. The light chains were all $\lambda$ type. Risk stratification resulted in 118 low-risk (34.0\%), 150 medium-risk (43.2\%), and 79 high-risk (22.8\%) patients.

Of 347 included patients, 79 received MDex, 103 received LDex, and 165 were treated by ASCT. Compared with the MDex or LDex groups, patients treated with ASCT had a more favorable baseline clinical manifestations, with a lower percentage of patients $>50$ years of age $(p=0.022)$, an extravascular water load $(p=0.001)$, or IgA type heavy chains $(p=0.048)$. Fewer high-risk patients were treated with ASCT than with MDex or LDex $(p<0.001)$. 
Table. 1 Baseline characteristics of POEMS syndrome patients

\begin{tabular}{|c|c|c|c|c|c|}
\hline Baseline charaterisitics & $\begin{array}{l}\text { All patients } \\
(N=347)\end{array}$ & $\begin{array}{l}\text { MDex } \\
(N=79)\end{array}$ & $\begin{array}{l}\text { ASCT } \\
(N=165)\end{array}$ & $\begin{array}{l}\text { LDex } \\
(N=103)\end{array}$ & $p$-value \\
\hline Age $>50$ years & 40.3 & 48.1 & 32.7 & 46.6 & 0.022 \\
\hline Male & 65.7 & 67.1 & 68.5 & 60.2 & 0.364 \\
\hline Polyneuropathy ONLS > 4 & 38.9 & 46.8 & 38.8 & 33.0 & 0.166 \\
\hline \multicolumn{6}{|l|}{ Organomegaly } \\
\hline Hepatomegaly & 38.3 & 43.0 & 40.6 & 31.1 & 0.238 \\
\hline Splenomegaly & 59.1 & 57.0 & 67.9 & 47.6 & 0.008 \\
\hline Lymphadenopathy & 64.0 & 69.6 & 60.6 & 65.0 & 0.376 \\
\hline IgA type heavy chain & 61.1 & 69.6 & 54.5 & 65.0 & 0.048 \\
\hline $\mathrm{SPE}>5 \mathrm{~g} / \mathrm{l}$ & 10.7 & 8.9 & 11.5 & 10.7 & 0.821 \\
\hline $\mathrm{BMPC}>10 \%$ & 3.7 & 1.3 & 4.8 & 3.9 & 0.441 \\
\hline Angioma & 59.4 & 55.7 & 52.1 & 68.9 & 0.062 \\
\hline Peripheral edema & 84.1 & 91.1 & 74.5 & 94.2 & 0.000 \\
\hline Ascites & 43.2 & 55.7 & 32.7 & 50.5 & 0.001 \\
\hline Pleural effusion & 37.8 & 50.6 & 29.1 & 41.7 & 0.003 \\
\hline Castleman disease $^{\mathrm{a}}$ & $61.5(N=52)$ & $41.7(N=12)$ & $65.5(N=29)$ & $2.7(N=11)$ & 0.249 \\
\hline Papilledema & 66.0 & 72.2 & 66.1 & 60.2 & 0.330 \\
\hline Osteosclerosis & 81.6 & 68.4 & 86.1 & 81.6 & 0.056 \\
\hline Hypoalbuminemia $(<30 \mathrm{~g} / \mathrm{l})$ & 13.0 & 19.0 & 10.3 & 12.6 & 0.167 \\
\hline Renal dysfunction $\left(\mathrm{eGFR}<30 \mathrm{ml} / \mathrm{min} / 1.73 \mathrm{~m}^{2}\right.$ ) & 4.6 & 5.1 & 3.6 & 5.8 & 0.691 \\
\hline $24 \mathrm{~h}$ urinary protein $>1 \mathrm{~g}$ & 5.8 & 5.1 & 4.2 & 7.8 & 0.482 \\
\hline VEGF $>4000 \mathrm{pg} / \mathrm{ml}$ & 57.6 & 41.8 & 58.8 & 67.0 & 0.004 \\
\hline \multicolumn{6}{|l|}{ Risk stratification } \\
\hline Low risk & 34.0 & 22.8 & 44.2 & 26.2 & 0.001 \\
\hline Medium risk & 43.2 & 43.0 & 43.0 & 42.7 & 0.994 \\
\hline High risk & 22.8 & 34.2 & 12.7 & 30.1 & 0.000 \\
\hline
\end{tabular}

The numbers were all presented as percentage

$A S C T$ autologous stem cell transplantation, Alb albumin, eGFR estimated glomerular filtration rate, $\operatorname{Ig} G$ immunoglobulin G, $O N L S$ Overall Neuropathy Limitations Scale, SPE serum protein electrophoresis, VEGF vascular endothelial growth factor

${ }^{a}$ Castleman's disease was diagnosed in 32 of 52 patients $(61.5 \%)$ who underwent tissue biopsies

\section{Treatment response}

Overall median follow-up was 45 months, total follow-up time was 1420 person-years. Median follow up was 90 months for MDex patients, 48 months for ASCT patients, and 44 months for LDex patients.

The total $\mathrm{CR}_{H}$ rate was $46.4 \%$. It was higher with ASCT (49.7\%) than with MDex $(37.7 \%, p=0.001)$. Subgroup analysis found that the regimen-associated difference was mainly in medium-high risk patients $(57.0 \%$ vs. $35.8 \%, p=$ 0.023 ), and the $\mathrm{CR}_{H}$ responses in the low-risk patients (40.6\% vs. $43.8 \%, p=1.000)$ were not significantly different. The $\mathrm{CR}_{H}$ rates in the LDex $(49.7 \%)$ and ASCT $(47.5 \%)$ groups were not significantly different $(p=0.797)$. (Fig. 1a).

The total $\mathrm{CR}_{V}$ rate was $55.1 \%$. $\mathrm{CR}_{V}$ was significantly better after ASCT than after MDex $(38.5 \%, p=0.001)$ or LDex $(47.7 \%, p=0.008)$. (Fig. 1a) Subgroup analysis found that the $\mathrm{CR}_{V}$ benefit of ASCT compared with the other regimens was significant in medium-high risk ( $p=$ $0.001)$, but not in low-risk ( $p=0.222)$. Moreover, $\mathrm{CR}_{V}$ was prognostic for PFS in medium-high risk patients, but not for OS in these groups. (Supplement Fig. 1).

Combined $\mathrm{CR}_{H}$ and $\mathrm{CR}_{V}$ have been associated with PFS and OS, and can be used as a surrogate endpoint [14]. The overall $\mathrm{CR}_{H} / \mathrm{CR}_{V}$ rate was $65.8 \%$ and the ASCT rate (75.3\%) was higher than that with MDex $(58.3 \%, p=$ $0.015)$ but not significantly different from the rate with LDex $(p=0.142)$. The difference in the $\mathrm{CR}_{H} / \mathrm{CR}_{V}$ rates in the ASCT and MDex groups was observed in medium-high risk patients $(p=0.001)$, but not in low-risk patients $(p=$ 0.748).

As for time to response, the overall median time to $\mathrm{CR}_{H}$ was 10 months (1-179 months). The median time to $\mathrm{CR}_{H}$ was 26.5 months (1-179 months) for MDex, 8.5 months (1-86 months) for ASCT and 7 months (1-46 months) for 

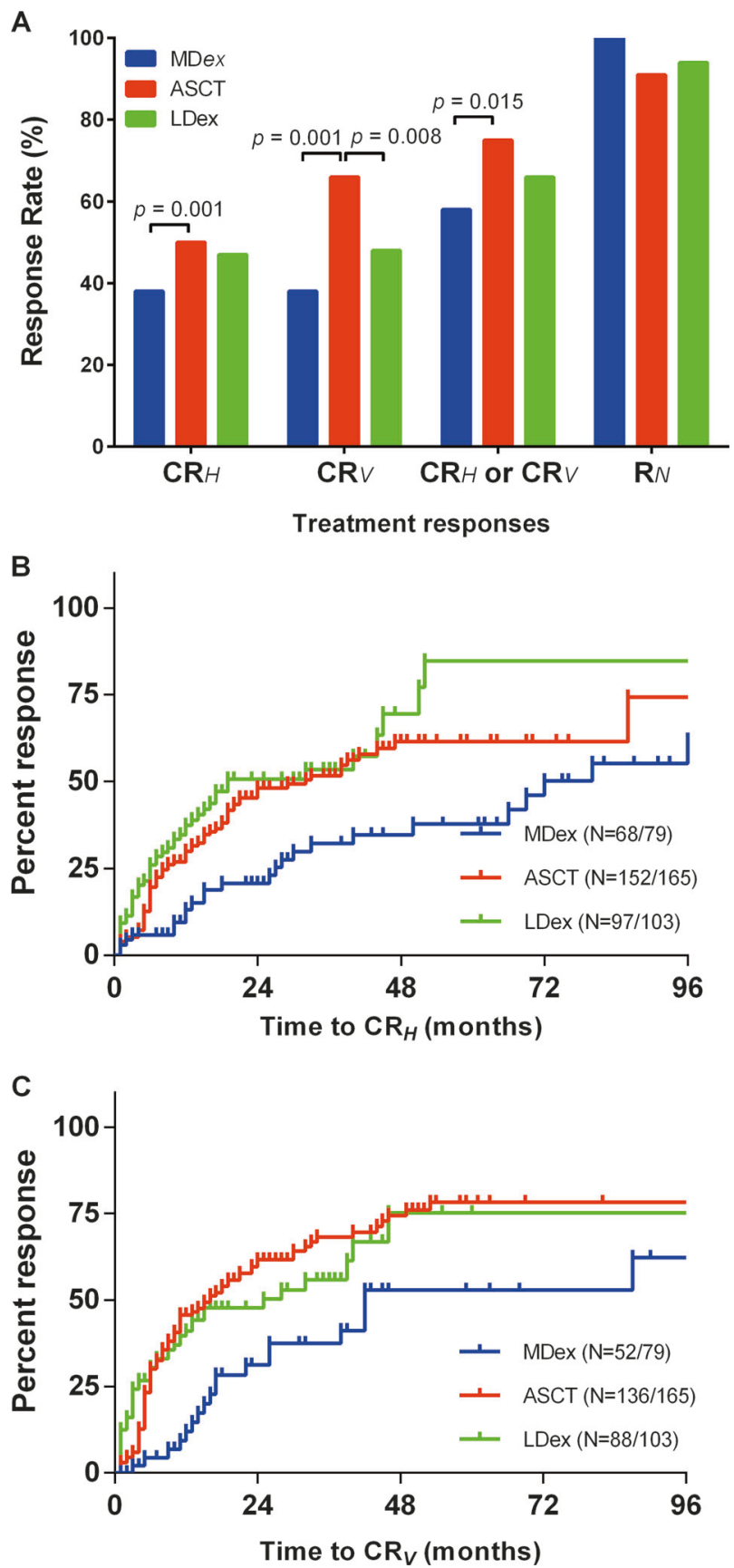

Fig. 1 Therapeutic efficacy and time to responses of ASCT, MDex, and LDex. a Hematologic complete remission, VEGF complete remission, and neurological remission rates. $\mathbf{b}$ Time to response for $\mathrm{CR}_{H}$. $\mathbf{c}$ Time to response for $\mathrm{CR}_{V}$

LDex, respectively. The total 6-months and 12-months $\mathrm{CR}_{H}$ rates were $19.2 \%$ and $26.1 \%$. The 6-months $\mathrm{CR}_{H}$ rate was $13.8 \%$ for ASCT, $5.9 \%$ for MDex and $21.4 \%$ for LDex. The 12-months $\mathrm{CR}_{H}$ rate was $27.9 \%$ for ASCT, $9.5 \%$ for MDex and $37.4 \%$ for LDex (Fig. 1b). The overall median time to $\mathrm{CR}_{V}$ was 8 months (1-163 months). The median time to $\mathrm{CR}_{V}$ was 17 months (1-163 months) for MDex, 8 months (1-140 months) for ASCT, and 4 months (1-46 months) for

Table. 2 Overall and risk subgroup analysis of best responses and survivals in three treatments

\begin{tabular}{|c|c|c|c|c|c|c|}
\hline \multirow[t]{2}{*}{ Treatment } & \multicolumn{2}{|c|}{$\begin{array}{l}\text { All } \\
(N=387)\end{array}$} & \multicolumn{2}{|c|}{$\begin{array}{l}\text { Low risk } \\
(N=118)\end{array}$} & \multicolumn{2}{|c|}{$\begin{array}{l}\text { Medium-high risk } \\
(N=229)\end{array}$} \\
\hline & Rate & $p$ & Rate & $p$ & Rate & $p$ \\
\hline \multicolumn{7}{|l|}{$\mathrm{CR}_{H}$} \\
\hline ASCT & 49.7 & & 40.6 & & 57.0 & \\
\hline MDex & 37.7 & 0.001 & 43.8 & 1.000 & 35.8 & 0.023 \\
\hline LDex & 47.5 & 0.797 & 48.0 & 0.638 & 47.3 & 0.267 \\
\hline \multicolumn{7}{|l|}{$\mathrm{CR}_{V}$} \\
\hline ASCT & 66.2 & & 62.1 & & 70.0 & \\
\hline MDex & 38.5 & 0.001 & 58.3 & 0.804 & 32.5 & $<0.001$ \\
\hline LDex & 47.7 & 0.008 & 41.7 & 0.097 & 50.0 & 0.022 \\
\hline \multicolumn{7}{|l|}{$\mathrm{CR}_{H} / \mathrm{CR}_{V}$} \\
\hline ASCT & 75.3 & & 70.0 & & 80.0 & \\
\hline MDex & 58.3 & 0.015 & 76.9 & 0.748 & 53.2 & 0.001 \\
\hline LDex & 66.3 & 0.142 & 56.0 & 0.204 & 70.1 & 0.167 \\
\hline \multicolumn{7}{|l|}{$\mathrm{R}_{N}$} \\
\hline ASCT & 91.5 & & 91.4 & & 91.5 & \\
\hline MDex & 100.0 & 0.188 & 100.0 & 1.000 & 100.0 & 0.295 \\
\hline \multirow[t]{2}{*}{ LDex } & 93.8 & 0.755 & 100.0 & 0.543 & 91.8 & 1.000 \\
\hline & $3 y$-PFS & $p$ & $3 y-P F S$ & $p$ & $3 y-P F S$ & $p$ \\
\hline \multicolumn{7}{|l|}{ PFS } \\
\hline ASCT & 87.6 & & 83.7 & 0.612 & 84.6 & \\
\hline MDex & 84.6 & 0.568 & 87.8 & 0.351 & 84.6 & 0.364 \\
\hline \multirow[t]{2}{*}{ LDex } & 64.9 & 0.003 & 59.0 & 0.016 & 67.7 & 0.037 \\
\hline & $3 y-O S$ & $p$ & $3 y-O S$ & $p$ & $3 y-O S$ & $p$ \\
\hline \multicolumn{7}{|l|}{ OS } \\
\hline ASCT & 94.4 & & 96.9 & & 92.3 & \\
\hline MDex & 90.7 & 0.088 & 94.3 & 0.701 & 89.8 & 0.155 \\
\hline LDex & 83.1 & 0.067 & 95.8 & 0.826 & 78.6 & 0.099 \\
\hline
\end{tabular}

LDex, respectively. The total 6-months and 12-months $\mathrm{CR}_{V}$ rates were $21.4 \%$ and $38.3 \%$. The 6-months $\mathrm{CR}_{V}$ rate was 23.8\% for ASCT, $7.2 \%$ for MDex, and $29.7 \%$ for LDex. The 12-months $\mathrm{CR}_{V}$ rate was $46.1 \%$ for ASCT, $16.1 \%$ for MDex and $37.7 \%$ for LDex (Fig. 1c).

The differences in $\mathrm{R}_{N}$ in the three treatment regimens, 100\% with MDex, 91.5\% with ASCT, and 93.8\% with LDex were not significant ( $p=0.234$ ) (Table 2).

\section{Survival}

Forty-nine of the 347 patients died during follow-up; two from lethal complications during ASCT. Fifty-one patients experienced disease progression and were alive at the end of the follow-up period. The overall 3-year PFS rate was $80.5 \%$ and was significantly higher in ASCT $(87.6 \%)$ than in LDex $(64.9 \%)$ patients $(p=0.003)$ but was not different from patients in the MDex group $(84.6 \%, p=0.568)$ (Fig. 2a). Subgroup analysis showed that PFS was longer with ASCT than with LDex in both low-risk $(p=0.016)$ and medium-high risk patients $(p=0.037)$ (Fig. 2c, d). 
A

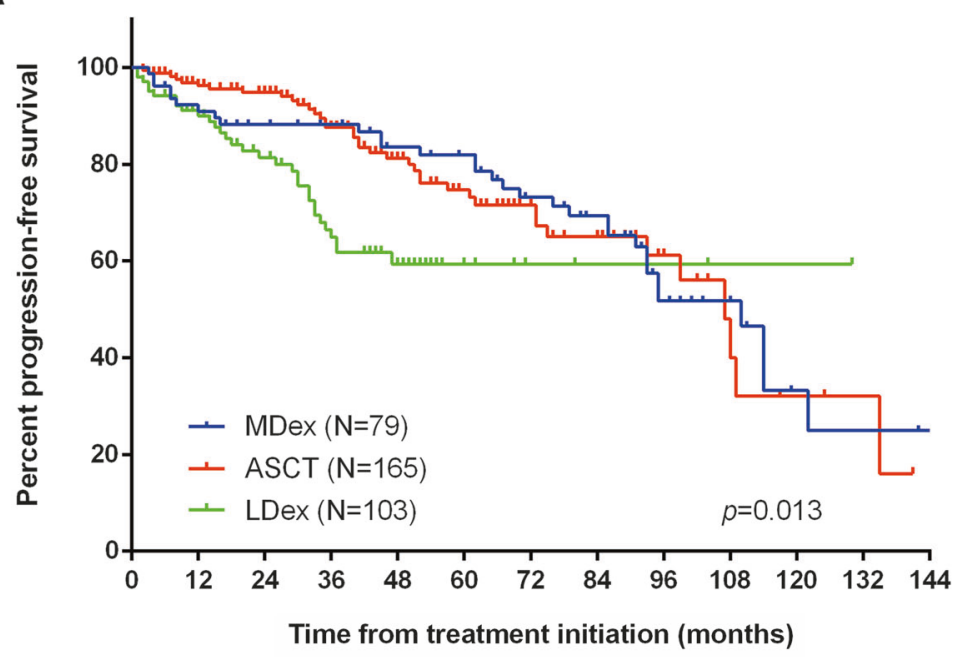

No. at risk:

$\begin{array}{lccccccccccccc}\text { MDex } & 79 & 69 & 61 & 55 & 52 & 46 & 32 & 27 & 14 & 7 & 3 & 3 & 2 \\ \text { ASCT } & 165 & 144 & 127 & 89 & 65 & 49 & 33 & 21 & 11 & 5 & 3 & 2 & 0 \\ \text { LDex } & 103 & 83 & 59 & 42 & 23 & 7 & 3 & 2 & 2 & 1 & 1 & 0 & 0\end{array}$

B

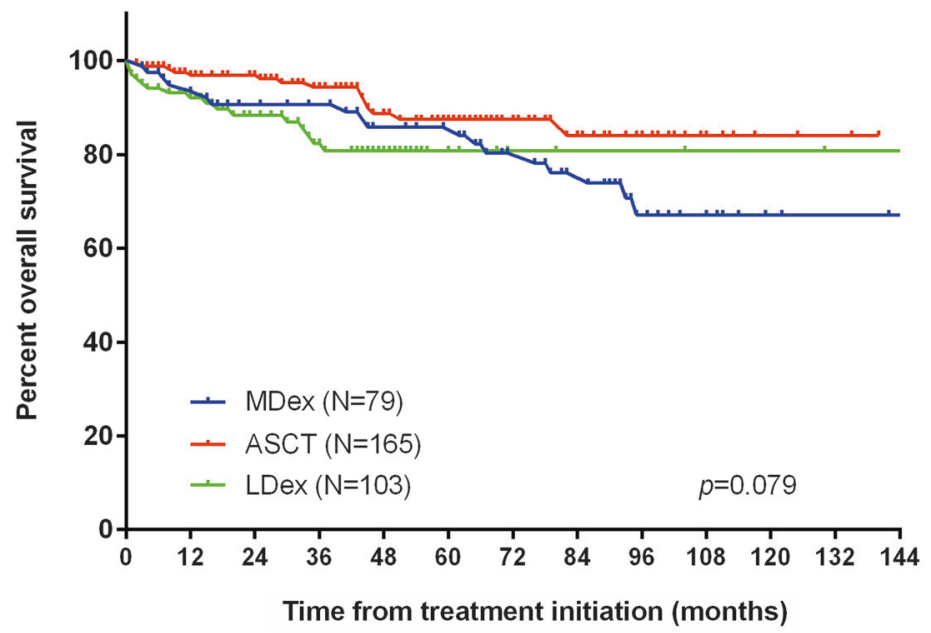

No. at risk:

$\begin{array}{lccccccccccccc}\text { MDex } & 79 & 69 & 61 & 58 & 52 & 48 & 39 & 34 & 16 & 10 & 4 & 3 & 2 \\ \text { ASCT } & 165 & 144 & 130 & 96 & 72 & 57 & 38 & 24 & 15 & 6 & 3 & 2 & 0 \\ \text { LDex } & 103 & 85 & 64 & 52 & 30 & 9 & 4 & 3 & 3 & 2 & 2 & 1 & 0\end{array}$

C

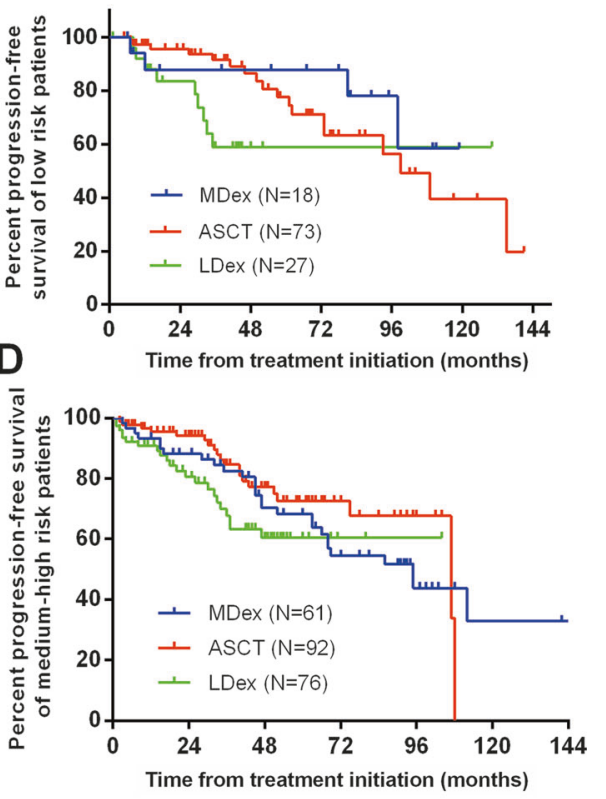

$\mathbf{E}$
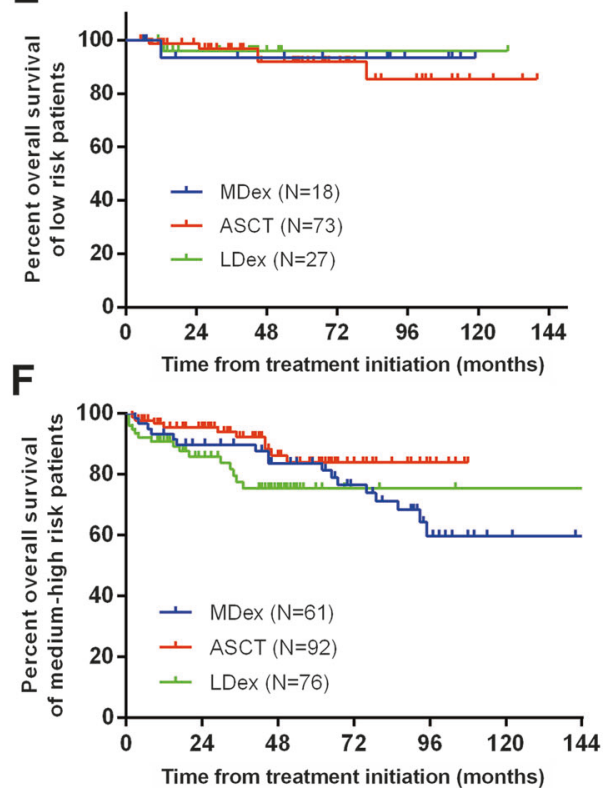

Fig. 2 Kaplan-Meier curves of OS and PFS of all, low-risk, mediumrisk, and high-risk patients treated with ASCT, MDex, or LDex (a) PFS in all patients, (b) OS in all patients, (c) PFS in low-risk patients, (d) PFS in medium-high risk patients, (e) OS in low-risk patients, and (f) OS in medium-high risk patients

\section{Discussion}

Overall 3-year OS was $90.4 \%$ and did not differ among the treatment groups $(94.4 \%$ vs. $90.7 \%$ vs. $83.1 \%, p=0.079)$ (Fig. 2b).

\section{Risk of secondary malignancy}

One secondary malignancy (hepatocellular carcinoma) was reported in the ASCT group. No secondary malignancies were reported in the LDex or MDex groups.
There are currently no standard treatments for patients with POEMS syndrome. ASCT, LDex, and MDex are all effective, but the question "what is the best treatment for POEMS syndrome?" has not been answered [12]. The rarity of POEMS syndrome makes it difficult to conduct randomized double-blind-controlled trials. Consequently, most have been retrospective analyses of single regimens or 
single-arm prospective studies. This retrospective analysis of 347 patients compared the three most common treatment options in POEMS syndrome, each with a different therapeutic basis. In this patient series, ASCT had the highest response and PFS rates of the three regimens, especially for patients at medium-to-high risk. One explanation is that ASCT eradicates the underlying plasma cell clones more completely than LDex or MDex. However, there were fewer high-risk patients and more patients with mild clinical manifestations in the ASCT group than in the MDex and LDex groups, which included patients with severe organ dysfunction. OS was not significantly better in ASCT patients than in the other groups, possibly because of treatment-related mortality $(1.2 \%)$, and long-term complications, such as secondary malignancy $(0.6 \%)$. On the other hand, OS can be improved with second-line treatments for patients with suboptimal responses. Good responses have been achieved with a wide range of treatment choices for refractory POEMS syndrome patients, which partly compensates for deficits in maintaining long-term PFS with first-line treatment. Salvage treatment with lenalidomide or bortezomib-based regimens have both shown promising response rates [15-17]. Novel regimens including daratumumab may further broaden the treatment options for multidrug-resistant patients [18].

MDex and LDex regimens have both been shown effective in POEMS syndrome patients not eligible for ASCT, but it is not clear that they are less toxic alternatives to ASCT as first-line POEMS therapy [8, 19]. In these patients, MDex regimens achieved response rates similar to ASCT in low-risk patients, but response was generally inferior in medium-high risk patients. However, long-term outcomes with MDex and ASCT were not significantly different. Melphalan therapy was generally not favored because of concerns of treatment-related stem cell damage, secondary myelodysplastic syndrome, and leukemia in myeloma patients, with a cumulative risk of 10-15\% [20, 21]. However, secondary malignancies or myelodysplastic syndrome were not reported in this patient series. The $3.2 \%$ occurrence of secondary malignancies reported in a Japanese cohort of POEMS patients was much lower than that seen in myeloma [2].

Lenalidomide was highly active in these newly diagnosed POEMS syndrome patients, with response rates and OS similar to those with ASCT. However, $\mathrm{CR}_{H}$ and $\mathrm{CR}_{H} /$ $\mathrm{CR}_{V}$ or after LDex treatment were not translated into longterm PFS. PFS after LDex treatment was inferior to that achieved with ASCT, even in low-risk patients. This may result from lack of maintenance treatment. Therefore, maintenance therapy was suggested for LDex group because limited cycles of LDex seemed insufficient to maintain long-term PFS compared with ASCT, though it remains to be shown whether adding maintenance treatment can increase PFS as did in myeloma patients [22].

In conclusion, all three treatments had reasonable responses and survivals in newly diagnosed POEMS syndrome patients. However, ASCT might have better responses and survivals compared with the other two groups, especially for higher risk patients. The treatment should be based on a comprehensive evaluation of the physical and the economic status of the patient, the planned follow-up, and the experience of the physicians and treatment center. ASCT could be recommended for high-risk patients if they are eligible. Lenalidomide maintenance after LDex treatment may be beneficial in term of PFS.

Acknowledgements The authors thank the patients and their families. Institutional research funding was provided by the National Natural Science Foundation of China (Grant No. 81570195), Beijing Natural Science Foundation (Grant No. 7182128), the Peking Union Medical College New Star (2011, to LJ), the CAMS Innovation Fund for Medical Sciences (Grant No. 2016-12M-1-002), and The National Key Research and Development Program of China (Grant No. 2016YFC0901503).

\section{Compliance with ethical standards}

Conflict of interest The authors declare that they have no conflict of interest.

Publisher's note: Springer Nature remains neutral with regard to jurisdictional claims in published maps and institutional affiliations.

Open Access This article is licensed under a Creative Commons Attribution 4.0 International License, which permits use, sharing, adaptation, distribution and reproduction in any medium or format, as long as you give appropriate credit to the original author(s) and the source, provide a link to the Creative Commons license, and indicate if changes were made. The images or other third party material in this article are included in the article's Creative Commons license, unless indicated otherwise in a credit line to the material. If material is not included in the article's Creative Commons license and your intended use is not permitted by statutory regulation or exceeds the permitted use, you will need to obtain permission directly from the copyright holder. To view a copy of this license, visit http://creativecommons. org/licenses/by/4.0/.

\section{References}

1. Dispenzieri A. POEMS syndrome: 2017 update on diagnosis, risk stratification, and management. Am J Hematol. 2017;92:814-29.

2. Kawajiri-Manako C, Sakaida E, Ohwada C, Miyamoto T, Azuma $\mathrm{T}$, Taguchi J, et al. Efficacy and long-term outcomes of autologous stem cell transplantation in POEMS syndrome: A Nationwide Survey in Japan. Biol Blood Marrow Transplant. 2018;24:1180-6.

3. Karam C, Klein CJ, Dispenzieri A, Dyck PJ, Mandrekar J, D'Souza A, et al. Polyneuropathy improvement following autologous stem cell transplantation for POEMS syndrome. Bone Marrow Transplant. 2015;84:1981-7.

4. D'Souza A, Lacy M, Gertz M, Kumar S, Buadi F, Hayman S, et al. Long-term outcomes after autologous stem cell transplantation for 
patients with POEMS syndrome (osteosclerotic myeloma): a single-center experience. Blood. 2012;120:56-62.

5. Dispenzieri A, Lacy MQ, Hayman SR, Kumar SK, Buadi F, Dingli D, et al. Peripheral blood stem cell transplant for POEMS syndrome is associated with high rates of engraftment syndrome. Eur J Haematol. 2008;80:397-406.

6. Li J, Duan MH, Wang C, Huang XF, Zhang W, Cao XX, et al. Impact of pretransplant induction therapy on autologous stem cell transplantation for patients with newly diagnosed POEMS syndrome. Leukemia. 2017;31:1375-81.

7. Jaccard A, Moreau P, Leblond V, Leleu X, Benboubker L, Hermine $\mathrm{O}$, et al. High-dose melphalan versus melphalan plus dexamethasone for AL amyloidosis. N Engl J Med. 2007;357:1083-93.

8. Li J, Zhang W, Jiao L, Duan MH, Guan HZ, Zhu WG, et al. Combination of melphalan and dexamethasone for patients with newly diagnosed POEMS syndrome. Blood. 2011;117:6445-9.

9. Kuwabara S, Misawa S, Kanai K, Suzuki Y, Kikkawa Y, Sawai S, et al. Neurologic improvement after peripheral blood stem cell transplantation in POEMS syndrome. Neurology. 2008;71:1691-5.

10. Dispenzieri A, Klein CJ, Mauermann ML. Lenalidomide therapy in a patient with POEMS syndrome. Blood. 2007;110:1075-6.

11. Li J, Huang XF, Cai QQ, Wang C, Cai H, Zhao $\mathrm{H}$, et al. A prospective phase II study of low dose lenalidomide plus dexamethasone in patients with newly diagnosed polyneuropathy, organomegaly, endocrinopathy, monoclonal gammopathy, and skin changes (POEMS) syndrome. Am J Hematol. 2018;93: 803-9.

12. Wang C, Huang XF, Cai QQ, Cao XX, Duan MH, Cai H, et al. Prognostic study for overall survival in patients with newly diagnosed POEMS syndrome. Leukemia. 2017;31:100-6.
13. Li J, Zhou DB. New advances in the diagnosis and treatment of POEMS syndrome. Br J Haematol. 2013;161:303-15.

14. Zhao H, Cai H, Wang C, Huang XF, Cao XX, Zhang L, et al. Prognostic value of serum vascular endothelial growth factor and hematological responses in patients with newly-diagnosed POEMS syndrome. Blood Cancer J. 2018;8:37.

15. Vannata B, Laurenti L, Chiusolo P, Sora F, Balducci M, Sabatelli $\mathrm{M}$, et al. Efficacy of lenalidomide plus dexamethasone for POEMS syndrome relapsed after autologous peripheral stem-cell transplantation. Am J Hematol. 2012;87:641-2.

16. Suichi T, Misawa S, Nagashima K, Sekiguchi Y, Shibuya K, Watanabe K, et al. Safety and efficacy of lenalidomide for intractable/recurrent poems syndrome: an open label pilot study. J Neurol Sci. 2017;381:1077.

17. Amino H, Misawa S, Sekiguchi Y, Suichi T, Kuwabara S. Safety and efficacy of bortezomib in patients with poems syndrome. J Neurol Sci. 2017;381(Suppl.):136-7.

18. Khan M, Stone K, van Rhee F. Daratumumab for POEMS Syndrome. Mayo Clin Proc. 2018;93:542-4.

19. Nozza A, Terenghi F, Gallia F, Adami F, Briani C, Merlini G, et al. Lenalidomide and dexamethasone in patients with POEMS syndrome: results of a prospective, open-label trial. Br J Haematol. 2017;179:748-55.

20. Rajkumar SV. Multiple myeloma: 2016 update on diagnosis, riskstratification, and management. Am J Hematol. 2016;91:719-34.

21. Yang J, Terebelo HR, Zonder JA. Secondary primary malignancies in multiple myeloma: an old NEMESIS revisited. Adv Hematol. 2012;2012:801495.

22. Attal M, Lauwers-Cances V, Marit G, Caillot D, Moreau P, Facon $\mathrm{T}$, et al. Lenalidomide maintenance after stem-cell transplantation for multiple myeloma. N Engl J Med. 2012;366:1782-91. 